\title{
FINITE MOMENT PROBLEMS AND APPLICATIONS TO MULTIPHASE COMPUTATIONS IN GEOMETRIC OPTICS*
}

\author{
LAURENT GOSSE ${ }^{\dagger}$ AND OLOF RUNBORG $\ddagger$
}

\begin{abstract}
Recovering a function out of a finite number of moments is generally an ill-posed inverse problem. We focus on two special cases arising from applications to multiphase geometric optics computations where this problem can be carried out in a restricted class of given densities. More precisely, we present a simple algorithm for the inversion of Markov's moment problem which appears in the treatment of Brenier and Corrias' "K-multibranch solutions" and study Stieltje's algorithm in order to process moment systems arising from a Wigner analysis. Numerical results are provided for moderately intricate wave-fields.
\end{abstract}

Key words. Moment problem, Vlasov equation, nonstrictly hyperbolic systems, geometric optics.

MSC subject classifications. 78A05, 35L65, 65M06

\section{Introduction}

1.1. Preliminaries: WKB ansatz for Schrödinger equation. We are interested in computing efficiently the high frequency asymptotics of the Cauchy problem for the following linear Schrödinger equation:

$$
i \varepsilon \partial_{t} \psi+\frac{\varepsilon^{2}}{2} \partial_{x x} \psi=0, \quad \psi(t=0, .)=\psi_{0} ; \quad x \in \mathbb{R} .
$$

The (small) dimensionless parameter $\varepsilon>0$ is usually called the scaled Planck constant and measures somehow the difference of magnitude between the characterisitic lengths of the physical process and the one of the quantum description of matter. In order to study the wave-particle transition (or classical limit), one is especially interested in a class of initial data of the $\mathrm{WKB}^{1}$ (or monokinetic) type:

$$
\psi(t=0, x)=A_{0}(x) \exp \left(i \varphi_{0}(x) / \varepsilon\right) ; \quad x \in \mathbb{R} .
$$

The slowly-varying quantities $A_{0} \geq 0$ and $\varphi_{0}$ appearing in this last expression are called respectively the amplitude and the phase of the wave $\psi_{0}$ which "looks like" a plane wave $a \exp (i b x / \varepsilon)$ on a finer scale $o(1)$. Data of the form (1.2) with $A_{0}$ compactly supported are usually called wave packets. WKB expansions are based on the assumption that for any value of the scaled Planck constant $\varepsilon$ below a certain threshold, the solution of (1.1)-(1.2) is well approximated by an ansatz,

$$
\psi(t, x) \simeq A(t, x) \exp (i \varphi(t, x) / \varepsilon), \quad t>0 .
$$

Interference phenomena generally require $A$ and $\varphi$ to be multivalued beyond some "breakup time". In this paper, we ignore phase shifts coming from Maslov indices

\footnotetext{
${ }^{*}$ Received: April 29, 2005; accepted (in revised version): June 1, 2005. Communicated by Shi Jin.

${ }^{\dagger}$ Corresponding Author, IAC-CNR "Mauro Picone" (sezione di Bari), Via Amendola 122/I -70126 Bari, Italy (l.gosse@ba.iac.cnr.it).

¥NADA, KTH, 10044 Stockholm, Sweden (olofr@nada.kth.se).

${ }^{1}$ After Wentzel, Krammers and Brillouin.
} 
produced by caustic crossing, in order to keep $A$ and $\varphi$ in $\mathbb{R}$. Plugging this ansatz and splitting between real and imaginary parts inside (1.1) leads to:

$$
\partial_{t} \varphi+\frac{1}{2}\left(\partial_{x} \varphi\right)^{2}=\frac{\varepsilon^{2}}{2 A} \partial_{x x} A, \quad \partial_{t}\left(A^{2}\right)+\partial_{x}\left(A^{2} \partial_{x} \varphi\right)=0
$$

The classical WKB system is formally obtained by letting $\frac{\varepsilon^{2}}{2 A} \partial_{x x} A \rightarrow 0$ when $\varepsilon \rightarrow 0$; see $[18,49]$ for details on this dispersive limit. Let us also recall that for small time, this kind of asymptotics are fully justified, see [26]. A survey is also given by Keller [33] and [4] proposes an interesting application to Quantum-Classical Molecular Dynamics (QCMD). In the limit, system (1.4) becomes weakly coupled as the eikonal equation decouples and can be solved independently; of course, one must give up the idea of solving in the context of viscosity solutions, $[22,37]$. If one introduces a velocity variable $u=\partial_{x} \varphi$, then the eikonal equation becomes the classical Burgers' equation

$$
\partial_{t} u+u \partial_{x} u=0, \quad u_{0}=\partial_{x} \varphi_{0},
$$

for which the multivalued (or geometric) solution is to be sought through the rays, $[9,30]$. If one can complete this program, then the intensity $A^{2}(t, x)$ can be easily recovered; indeed, in the homogeneous case and for any time $t>0$, one gets:

$$
A^{2}(t, x)=A_{0}^{2}(y)\left|\frac{\partial y}{\partial x}\right|, \quad x=y+t u_{0}(y)=y+t u(t, x) .
$$

Hence a convenient and accurate way to derive the intensity follows from

$$
\left|\frac{\partial y}{\partial x}\right|=\left|\frac{\partial x}{\partial y}\right|^{-1}=\frac{1}{\left|1+t u_{0}^{\prime}(y)\right|} \quad \Rightarrow \quad A^{2}(t, x)=\frac{A_{0}(y)^{2}}{\left|1+t u_{0}^{\prime}(y)\right|}
$$

It has already been observed in [9] that the geometric solution to (1.5) when $u_{0} \geq 0$ is given exactly by one of the free transport equations

$$
\partial_{t} f+\xi \partial_{x} f=0, \quad f(t=0, x, \xi)=H\left(u^{0}(x)-\xi\right) H(\xi),
$$

where $H$ stands for the Heaviside function. But, as soon as $u_{0}$ is not everywhere increasing, folds develop after some (finite) time and a correct expression for $f$ reads:

$$
f(t, x, \xi)=\sum_{k=1}^{K(t)}(-1)^{k-1} H\left(u_{k}(t, x)-\xi\right), \quad u_{k}>u_{k+1},
$$

where $K(t)$ is the number of branches $u_{k}$ showing up in $u$. The remarkable observation in $[10,11]$ is that in case $K(t) \leq K \in \mathbb{N}$, the exact solution of $(1.7)$ can be recovered at any time $t>0$ from a $K \times K$ hyperbolic moment system. Relying on an entropy minimization principle, it is theoretically possible to express the $K+1^{\text {th }}$ moment knowing the $K$ preceding ones.

A feasible route for computing moderately intricate WKB ansatz in a onedimensional framework is to solve

$$
\partial_{t} \vec{m}+\partial_{x} F_{K}(\vec{m})=0
$$


where

$$
\vec{m}=\left(\begin{array}{c}
m_{1} \\
\vdots \\
m_{K}
\end{array}\right), \quad F_{K}(\vec{m})=\left(\begin{array}{c}
m_{2} \\
\vdots \\
m_{K+1}
\end{array}\right), \quad m_{j}=\int_{0}^{X} \xi^{j-1} f(t, x, \xi) d \xi=\frac{1}{j} \sum_{k=1}^{K}(-1)^{k-1} u_{k}^{j} .
$$

for some $X>u_{1}$. This follows from multiplying (1.7) by $\xi^{j}$, integrating in $\xi$ over $[0, X]$ and using the closure assumption (1.8). It is generally impossible to derive a closed form expression for the last component of $F_{K}$ in terms of $\vec{m}$ when $K>4$ (see however [46] in case $K \leq 4)$; a convenient way out is thus to invert the mapping $\vec{u} \mapsto \vec{m}$ and then take advantage of the preceding expressions. This paper focuses therefore on computational aspects of the (inverse) moment problems in the context of densities (1.8).

The presentation here follows computations of semiclassical approximations to Schrödinger's equation (1.1); however, adapting to other geometric optics frameworks is merely straightforward as the recovery of the $u_{k}$ 's from the $m_{k}$ 's in (1.10) opens the way to computing any nonlinear function $\tilde{F}_{K}(\vec{m})$, see for instance the example in [24].

1.2. Classical results on the infinite moment problem. The general form is Hausdorff's one; namely, let $m_{1}, m_{2}, \ldots$ be an infinite sequence of real numbers. When is there a positive bounded measure $\mu \in \mathcal{M}^{+}(0,1)$ such that:

$$
m_{j}=\int_{0}^{1} \xi^{j-1} \cdot \mu(d \xi), \quad j \in \mathbb{N} ?
$$

The solution of this classical problem goes as follows: let $\Delta t_{j} \stackrel{\text { def }}{=} t_{j+1}-t_{j}$ stand for the forward difference operator. One defines the infinite triangular array:

$$
s_{i, j}=(-1)^{i-j}\left(\begin{array}{c}
i \\
j
\end{array}\right) \Delta^{i-j} m_{j}, \quad\left(\begin{array}{l}
i \\
j
\end{array}\right)=\frac{i !}{j !(i-j) !},
$$

$j \leq i \in \mathbb{N}, \Delta^{0} m_{j}=m_{j}$ and $\Delta^{i+1} m_{j}=\Delta^{i} m_{j+1}-\Delta^{i} m_{j}$. From [15], we state:

THEOREM 1.1. Let $\left(m_{k}\right)_{k \in \mathbb{N}}$ be a given sequence and define the auxiliary array (1.12). There exists a positive measure $\mu$ on $[0,1]$ such that (1.11) holds if and only if $\left(s_{i, j}\right)_{i \geq j} \in \mathbb{R}^{+}$; moreover $\mu$ is unique.

The Markov's moment problem is the restriction to positive measures having uniformly bounded densities: $\mu(d \xi)=f(\xi) . d \xi$, with $|f(\xi)| \leq C \in \mathbb{R}^{+}$.

Theorem 1.2. Let $C \in \mathbb{R}^{+}$and $\left(m_{k}\right)_{k \in \mathbb{N}}$. There exists a $\mu \in \mathcal{M}^{+}(0,1)$ such that

(i) (1.11) holds,

(ii) $\mu$ is absolutely continuous,

(iii) $|d \mu / d \xi| \leq C$ almost everywhere,

if and only if for all $i \geq j, 0 \leq s_{i, j} \leq \frac{C}{i+1}$; moreover $\mu$ is unique.

These results state existence and uniqueness of the solution in case one has an infinite number of moment values as an input. However, this situation is not common in practical problems. Hence some investigations have been devoted to the inversion of finite problems; for instance, [50] proposes an algorithm to treat Hausdorff's problem with $n \in\{1,2, \ldots, 2 K\}$ (see also [28,29]). A common approach to restore stability in 
Markov's problem is via entropy optimization; consult e.g. [5, 6, 39, 43]. We refer to $[15,38]$ for some historical background.

The paper is organized as follows: $\S 2$ summarizes several results on the finite Markov problem. $\S 3$ is devoted to the study of a rather simple inversion algorithm for those problems. $\$ 4$ deals briefly with a more singular closure based on Wigner analysis and $\S 5$ is concerned with numerical experiments.

\section{Several variants of Markov's moment problem}

In this section, we enumerate several ways to solve the finite Markov moment problem for a kinetic density $0 \leq f(\xi) \leq 1$, including the classical entropy minimization process, and state equivalence results. This will allow us to choose among them an efficient algorithm for our numerical purposes.

We follow [47] and recall a definition of the Markov's moment problem on the smallest interval of $\mathbb{R}^{+}$denoted hereafter by $[0, X], X \in \mathbb{R}^{+}$. We assume that we are given a collection of values $\vec{m}=\left(m_{k}\right)_{k=1, \ldots, K} \in \mathbb{R}^{K}$ and seek a bounded measurable density function such that:

$$
\int_{\mathbb{R}^{+}} \xi^{k-1} f(\xi) \cdot H(X-\xi) \cdot d \xi=m_{k}, \quad \forall k=1, \ldots, K,
$$

where $H$ stands for the classical Heaviside function. Of course, one necessary condition for such a solution to exist is for the moments $m_{k}$ 's to be realizable (one also speaks about extendable vectors of $\mathbb{R}^{K}$ ), i.e. that $\exists f \in L^{\infty}$ such that (2.1) holds.

Generally, the finite set of conditions (2.1) alone do not determine uniquely a kinetic density $f$ and we will now go through several ways to augment the problem in order to have a unique and computable solution.

2.1. The min-power problem on $\mathbb{R}^{+}$. The min-power version of Markov's moment problem is given by the following definition:

Definition 2.1. (Sklyar, Fardigola, [47]) Let $K \in \mathbb{N}$; a pair $(f, X) \in L^{\infty} \times \mathbb{R}^{+}$is a solution of the min-power problem (2.1) if and only if the following three conditions hold:

(i) for $(f, X)$, the equalities (2.1) are valid,

(ii) $|f(\xi)|=1$ almost everywhere on $] 0, X[$,

(iii) $f$ has no more than $K-1$ discontinuity points inside $] 0, X[$.

For this problem it has been proposed in [47] a constructive proof of the existence of a solution $(f, X)$ of (2.1) satisfying (i)-(iii) under some mild assumptions on the values $m_{k}$ 's. The authors state also that the solution built in this way is unique; it reads

$$
f(\xi)=\alpha \sum_{k=1}^{K}(-1)^{k+1}\left(H\left(\xi-x_{k-1}\right)-H\left(\xi-x_{k}\right)\right),
$$

where $\alpha= \pm 1$ and $0=x_{0} \leq x_{1} \leq \ldots \leq x_{K}=X$. It can be noted this solution $f$ has at most $K$ discontinuity points on $\mathbb{R}^{+}$. The construction also leads to a "density function" taking values inside $\{-1,0,1\}$.

The recursive algorithm proposed in [47] to compute $\left\{u_{j}\right\}$ is, however, not suitable for direct numerical implementation. In the next section we will discuss a way to simplify it in order to use it together with a PDE solver for (1.9). 
2.2. Entropy minimization criterion on $\mathbb{R}^{+}$. A common way to restore uniqueness in the inverse problem (2.1) is to minimize a strictly convex entropy function $\phi(f)$ :

$$
J_{\phi}^{K}(\vec{m})=\inf _{f}\left\{\int_{\mathbb{R}^{+}} \phi(f)(\xi) \cdot d \xi ; \quad \int_{\mathbb{R}^{+}} \xi^{k-1} f(\xi) \cdot d \xi=m_{k}, \quad k=1, \ldots, K\right\} .
$$

Classical results on this problem in statistical mechanics involve for instance Boltzmann, Shannon or Fermi-Dirac's ones; consult [6]. A formal argument to solve (2.3) relying on Lagrange's multipliers goes as follows: we introduce the $K$ multipliers $\lambda_{k}$ for the constraints (2.1) and rewrite (2.3) as

$$
J_{\phi}^{K}(\vec{m})=\inf _{f}\left\{\int_{\mathbb{R}^{+}} \phi(f)(\xi) \cdot d \xi-\sum_{k=1}^{K} \lambda_{k}\left(\int_{\mathbb{R}^{+}} \xi^{k-1} f(\xi) \cdot d \xi-m_{k}\right)\right\} .
$$

In case $\phi$ is strictly convex, $\phi^{\prime}$ is invertible and one may "differentiate" with respect to $f$ in order to derive, for some $h(\xi)$,

$$
\int_{\mathbb{R}^{+}}\left(\phi^{\prime}(f)(\xi)-\sum_{k=1}^{K} \lambda_{k} \xi^{k-1}\right) h(\xi) \cdot d \xi,
$$

which leads to the well-known expression $f(\xi)=\left(\phi^{\prime}\right)^{-1}\left(\sum_{k=1}^{K} \lambda_{k} \xi^{k-1}\right)$. Indeed, this result has been obtained rigorously by the authors of [6] relying on Fenchel's duality theory. Notice a difference with the min-power problem since the moment equalities (2.1) represent only a constraint in the convex programming problem (2.3).

In their paper [11], Brenier and Corrias set up a similar program to derive the so-called " $K$-branch Maxwellian" to be used in their kinetic formulation of " $K$ multibranch solutions" of Burgers' equation. They consider a set of linear entropies inside (2.3). More precisely,

$$
\phi_{\theta}(f)(\xi)=\theta(\xi) \cdot f(\xi) ; \quad \alpha \leq \partial_{\xi}^{K} \theta(\xi) \leq \frac{1}{\alpha}, \quad \alpha \in \mathbb{R}_{*}^{+} .
$$

This choice is well-suited for the derivation of kinetic formulations via BGK approximation. We remark that the set of entropies can also be made nonlinear by including the constraint by means of $\phi_{\infty}(f)=\left\{\begin{array}{c}0 \text { if } 0 \leq f \leq 1 \\ +\infty \text { otherwise. }\end{array}\right.$

Using again Fenchel's duality theorem, they established existence and uniqueness of the solution of (2.3) in this context; moreover it does not depend on the choice of $\theta$. We can see that our former (formal) argument leaves us with

$$
J_{\theta}^{K}(\vec{m})=\inf _{f}\left\{\int_{\mathbb{R}^{+}}\left(\theta(\xi)-\sum_{k=1}^{K} \lambda_{k} \xi^{k-1}\right) f(\xi) \cdot d \xi+\sum_{k=1}^{K} \lambda_{k} m_{k}\right\} .
$$

Since $f \geq 0$, in order to achieve a minimum of this functional, the optimal $f$ can be expected to be supported only on the intervals of $\mathbb{R}^{+}$where the polynomial function $\xi^{K}-\sum_{k=1}^{K} \lambda_{k} \xi^{k-1}$ is negative. This is indeed the case and we can now recall part of the main result in [11]:

Theorem 2.1. (Brenier $\&$ Corrias, [11]) Let $K \in \mathbb{N}$ and $\vec{m}=\left(m_{k}\right)_{k=1, \ldots, K}$ be a sequence of realizable moments; there exists a unique $f$ solution of (2.3)-(2.4) for all admissible functions $\theta$ and characterized by the equivalent properties: 
(i) for at least one $\theta$, there exist $\left(\lambda_{k}\right)_{k=1, \ldots, K} \in \mathbb{R}^{K}$ such that $f(\xi)=$ $H\left(-\theta(\xi)+\sum_{k=1}^{K} \lambda_{k} \xi^{k-1}\right)$,

(ii) for each admissible $\theta$, there exist such a vector $\left(\lambda_{k}\right)_{k=1, \ldots, K} \in \mathbb{R}^{K}$ and (2.3)(2.4) is equivalent to the finite dimensional dual problem:

$$
J_{\theta}^{K}(\vec{m})=\sup _{\vec{\lambda} \in \mathbb{R}^{K}}\left\{\sum_{k=1}^{K} \lambda_{k} m_{k}-\int_{\mathbb{R}^{+}} \max \left(0, \theta(\xi)-\sum_{k=1}^{K} \lambda_{k} \xi^{k-1}\right) \cdot d \xi\right\} .
$$

If all the $u_{k}$ 's are real, one can write $f(\xi)=H\left(-\prod_{k=1}^{K}\left(\xi-u_{k}\right)\right) \in\{0,1\}$. In order to compare with (2.2), let us have a closer look at this unique solution to (2.3)-(2.4):

- (i) says exactly that $f$ is the characteristic function of a collection of intervals $\left[u_{k+1}, u_{k}\right]$ where the $u_{k}$ 's are roots of $\xi^{K}-\sum_{k=1}^{K} \lambda_{k} \xi^{k-1}=0$, with the convention $u_{k+1}<u_{k}$ for $k=1, \ldots, K$.

- $f$ has the same structure as the solution of (2.1) in the sense of Def. 2.1 on the interval $] 0, u_{1}\left[\left(\right.\right.$ i.e. $X=u_{1}$ in $\left.(2.1)\right)$ and it has $K$ discontinuity points on $\mathbb{R}^{+}$. It is not known whether (2.2) optimizes a certain entropy function.

2.3. $L^{\infty}$ moment problem on an interval. A third way to invert the set of $K$ equations (2.1) in a unique and stable way is to try to minimize the $L^{\infty}$ norm of $f$; this is the approach developed in [40,44]. Namely, one completes $(2.1)$ as follows, for some given $X \in \mathbb{R}^{+}$,

$$
I_{\infty}^{K}(\vec{m})=\inf _{f}\left\{\|f\|_{L^{\infty}}, \quad f \geq 0, \quad \int_{0}^{X} \xi^{k-1} f(\xi) \cdot d \xi=m_{k}, \quad k=1, \ldots, K\right\} .
$$

This problem may also be treated including convenient lattice bounds, namely $\alpha(\xi) \leq$ $f(\xi) \leq \beta(\xi)$, see [44]. In this context, the Lagrange multipliers technique does not make sense at all since the $L^{\infty}$ norm is not differentiable. However, it has been shown in [44] that (2.6) is equivalent to the linear problem:

$$
I_{\infty}^{K}(\vec{m})=\inf _{f, q}\left\{q,(f, q) \in L^{\infty} \times \mathbb{R}^{+}, 0 \leq f \leq q, \quad \int_{0}^{X} \xi^{k-1} f(\xi) . d \xi=m_{k}, \quad k=1, \ldots, K\right\} .
$$

Chapter 4 of [44] is devoted to a proof of existence and uniqueness of the optimal solution to (2.7) using again Fenchel's duality theory. This means also that the optimal solution to (2.6) matches the one of (2.7). Moreover, the optimal $f$ has the following form:

$$
f(\xi)=\|f\|_{L^{\infty}} H\left(\sum_{k=1}^{K} \lambda_{k} \xi^{k-1}\right)=\|f\|_{L^{\infty}} H\left(a \prod_{k=2}^{K}\left(\xi-u_{k}\right)\right) ; \quad \lambda_{k} \in \mathbb{R}^{K}, a \in \mathbb{R} .
$$

So in case $\|f\|_{L^{\infty}}=1$, by uniqueness it matches the " $K$-branch Maxwellian" in case $X=u_{1}$. Our main interest in this approach is that the linear programming problem (2.7) can be easily implemented via the standard Simplex method. However, we found that even if linear programming can represent a very reliable technique to compute the last component of $F_{K}(\vec{m})(1.10)$, it is generally unstable for the computation of the K values $u_{1}, \ldots, u_{K}$. This is illustrated on the elementary case $K=3$ while considering the equation (1.5) on $x \in[0,2 \pi]$ with initial data $u_{0}(x)=\frac{1}{2}\left(0.5+\exp \left(-2(x-\pi / 2)^{2}\right)\right)$; see Fig.2.1. We stress that in order to produce these results, inverting Markov moments 

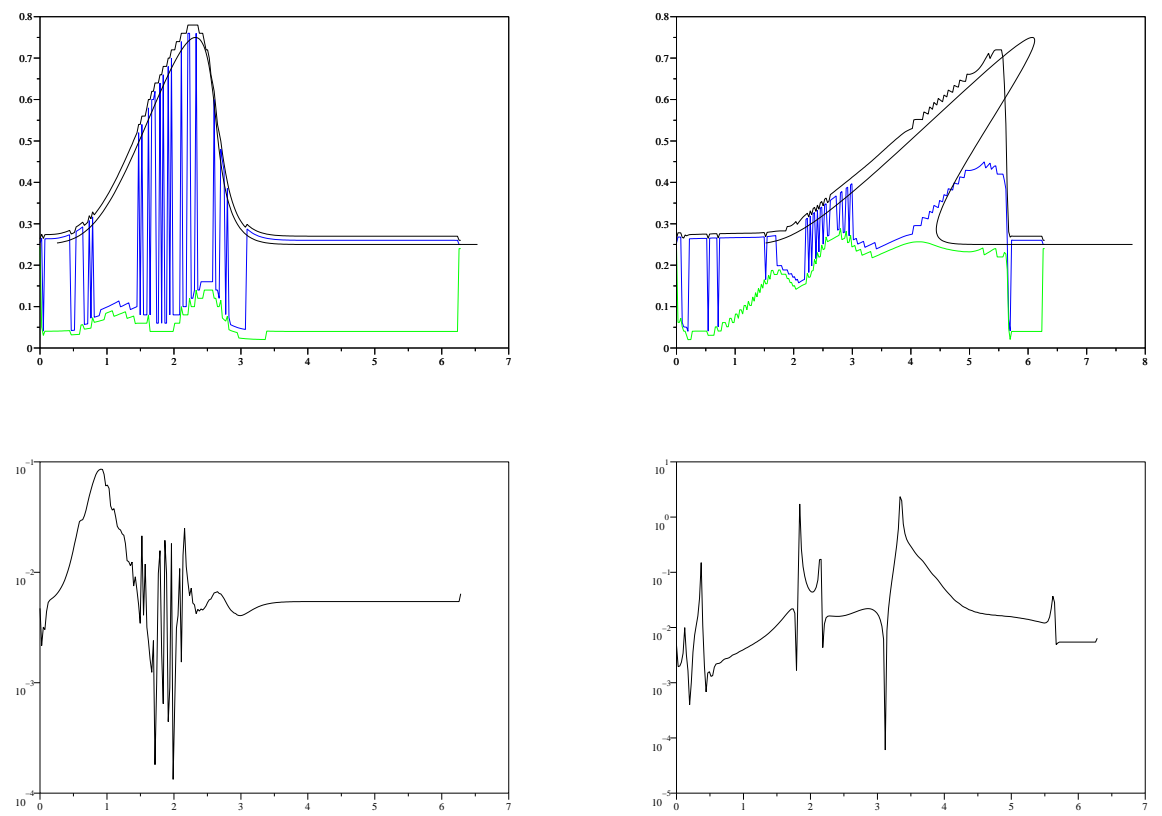

FIG. 2.1. Comparison between 3-branch solutions (top, solid line is exact solution) and the absolute error on the 4 th moment (bottom) for the Simplex method at $t=1$ (left) and $t=6$ (right).

(1.10) inside each computational cell, in each timestep, is necessary, hence the need to perform this task in the most stable way.

Another important point in [40], is a very strong stability result, the so-called "superresolution" of Markov's moment problem:

Theorem 2.2. (Lewis, [40]) Suppose $f(\xi)=H\left(\sum_{k=1}^{K} \lambda_{k} \xi^{k-1}\right)$ for some $\vec{\lambda} \in \mathbb{R}^{K}$ with $\sum_{\text {if }}^{K} k=1 \lambda_{k} \xi^{k-1} \neq 0$ a.e. Then for any sequence of measurable functions $f^{\varepsilon}:[0, X] \mapsto[0,1]$,

$$
\int_{0}^{X} \xi^{k-1} f^{\varepsilon}(\xi) \cdot d \xi \rightarrow m_{k}, \quad k=1, \ldots, K,
$$

then it follows that $\left\|f^{\varepsilon}-f\right\|_{L^{1}(0, X)} \rightarrow 0$. Under mild additional assumptions, an error estimate is furthermore derived:

$$
\left\|f^{\varepsilon}-f\right\|_{L^{1}(0, X)} \leq O(1)\left\|\int_{0}^{X}\left(1 \xi \ldots \xi^{K-1}\right)^{T} f^{\varepsilon}(\xi) . d \xi-\vec{m}\right\|^{\frac{1}{2}} .
$$

(See also [46], Sec. 3.1 for a similar result.) This suggests that even if the linear programming approach does not work well, hopefully another numerical method could allow us to invert Markov's moments since the problem is shown to be strongly stable in the sense that the errors in $\left\{u_{k}\right\}$ will be of the same order as the errors in the moments $\left\{m_{k}\right\}$. Typically, errors generated by numerical approximations of hyperbolic systems are of the order $\sqrt{\Delta x}$, where $\Delta x$ is the grid size, see $[20,46]$. 


\section{An efficient algorithm to invert Markov's moments}

In this section, we recall briefly the algorithm originally introduced in [34, 47, 48] in order to invert the min-power Markov moment problem. The original algorithm is not directly suitable for computational purposes, having too high complexity and unclear stability properties. We propose here a simplification which leads subsequently to a much lighter version, involving the solution of a well-conditioned triangular linear system of equations and a generalized eigenvalue problem. In a forthcoming paper [25] we shall give a direct proof that the simplified algorithm works, and discuss its stability.

3.1. The original Korobov-Sklyar algorithm for $K \in 2 \mathbb{N}$. Recall first the expressions (1.10). The goal is to extract $\left\{u_{k}\right\}$ given $\left\{m_{k}\right\}, k=1, \ldots, K$. We show now the original algorithm, modified to the case when $f \in\{0,1\}$ rather than $f \in\{-1,1\}$.

Start by defining the polynomials

$$
U_{k}(u)=u^{k}-k m_{k}, \quad V_{k}(u)=u^{k}+k m_{k},
$$

and set $P_{1}=U_{1}, Q_{1}=V_{1}$. Then compute repeatedly for $k=2, \ldots, K$,

$$
P_{k}(u)=\frac{1}{k}\left(U_{k}(u)-\sum_{\ell=1}^{k-1} U_{\ell}(u) P_{k-\ell}(u)\right), \quad Q_{k}(u)=\frac{1}{k}\left(V_{k}(u)-\sum_{\ell=1}^{k-1} V_{\ell}(u) Q_{k-\ell}(u)\right) .
$$

Afterwards, the following (polynomial) matrices are built:

$$
\mathbf{P}(u)=\left(\begin{array}{cccc}
P_{2} & P_{3} & \ldots & P_{1+\frac{K}{2}} \\
P_{3} & P_{4} & \ldots & P_{2+\frac{K}{2}} \\
\vdots & \vdots & \ddots & \vdots \\
P_{1+\frac{K}{2}} & P_{2+\frac{K}{2}} & \ldots & P_{K}
\end{array}\right), \quad \mathbf{Q}(u)=\left(\begin{array}{cccc}
Q_{2} & Q_{3} & \ldots & Q_{1+\frac{K}{2}} \\
Q_{3} & Q_{4} & \ldots & Q_{2+\frac{K}{2}} \\
\vdots & \vdots & \ddots & \vdots \\
Q_{1+\frac{K}{2}} & Q_{2+\frac{K}{2}} & \ldots & Q_{K}
\end{array}\right)
$$

They finally generate the polynomial $\Pi(u)=\operatorname{det}(\mathbf{P})(u) \cdot \operatorname{det}(\mathbf{Q})(u)$; the largest root of $\Pi$ is the largest value among the $\left\{u_{k}\right\}$, thus $u_{1}$. Next, the moments are modified by subtracting off the term involving $u_{1}$, letting $\tilde{m}_{j}=m_{j}-u_{1}^{j} / j$. The procedure is then applied to $\tilde{m}_{j}$, and repeated recursively until all values are found.

3.2. The simplified algorithm: even case $(K \in 2 \mathbb{N})$. Our first observation is that the roots of $\Pi(u)$ are in fact precisely the values that we are looking for. Moreover, the $P_{k}$ and $Q_{k}$ previously defined are just first degree polynomials as we show now. For notational simplicity, we redefine the moments $m_{k}$ as follows:

$$
m_{j}=\sum_{k=1}^{K}(-1)^{k-1} u_{k}^{j}
$$

Lemma 3.1. The polynomials $P_{k}, Q_{k}$, (3.1) belong to $\mathbb{P}_{1}(\mathbb{R})$ for $k=1, \ldots, K \in 2 \mathbb{N}$ :

$$
\begin{cases}P_{k}(u)=a_{k-1} u-a_{k} \quad \text { with } \quad a_{k}=\frac{1}{k} \sum_{\ell=1}^{k} m_{\ell} a_{k-\ell}, & a_{0}=1 \\ Q_{k}(u)=b_{k-1} u-b_{k} \quad \text { with } \quad b_{k}=-\frac{1}{k} \sum_{\ell=1}^{k} m_{\ell} b_{k-\ell}, & b_{0}=1 .\end{cases}
$$


Proof. We proceed by induction; the property is clearly true for $k=1$ since $P_{1}(u)=$ $V_{1}(u)=u-m_{1}$. Let us suppose that the formulas hold for all $k$ up to some $k=n \in \mathbb{N}$. We then get for $k=n+1$,

$$
\begin{aligned}
& P_{n+1}(u) \\
= & \frac{1}{n+1}\left(U_{n+1}(u)-\sum_{k=1}^{n} U_{k}(u) P_{n+1-k}(u)\right) \\
= & \frac{1}{n+1}\left(u^{n+1}-m_{n+1}-\sum_{k=1}^{n}\left(u^{k}-m_{k}\right)\left(a_{n-k} u-a_{n+1-k}\right)\right) \\
= & \frac{1}{n+1}\left(u^{n+1}-m_{n+1}-\sum_{k=1}^{n} a_{n-k} u^{k+1}-a_{n+1-k} u^{k}-m_{k} a_{n-k} u+m_{k} a_{n+1-k}\right) \\
= & \frac{1}{n+1}\left(u^{n+1}-m_{n+1}-a_{0} u^{n+1}+a_{n} u+\sum_{k=1}^{n}\left(m_{k} a_{n-k} u-m_{k} a_{n+1-k}\right)\right) \\
& \left(\operatorname{because} a_{0}=1\right) \\
= & \frac{1}{n+1}\left(\left(a_{n}+\sum_{k=1}^{n} m_{k} a_{n-k}\right) u-\left(m_{n+1}+\sum_{k=1}^{n} m_{k} a_{n+1-k}\right)\right) \\
= & a_{n} u-\frac{1}{n+1} \sum_{k=1}^{n+1} m_{k} a_{n+1-k},
\end{aligned}
$$

and one identifies

$$
a_{n+1}=\frac{1}{n+1} \sum_{k=1}^{n+1} m_{k} a_{n+1-k} .
$$

The procedure also applies for

$$
\begin{aligned}
& Q_{n+1}(u) \\
= & \frac{1}{n+1}\left(V_{n+1}(u)-\sum_{k=1}^{n} V_{k}(u) Q_{n+1-k}(u)\right) \\
= & \frac{1}{n+1}\left(u^{n+1}+m_{n+1}-\sum_{k=1}^{n}\left(u^{k}+m_{k}\right)\left(b_{n-k} u-b_{n+1-k}\right)\right) \\
= & \frac{1}{n+1}\left(u^{n+1}+m_{n+1}-\sum_{k=1}^{n} b_{n-k} u^{k+1}-b_{n+1-k} u^{k}+m_{k} b_{n-k} u-m_{k} b_{n+1-k}\right) \\
= & \frac{1}{n+1}\left(u^{n+1}+m_{n+1}-b_{0} u^{n+1}+b_{n} u-\sum_{k=1}^{n}\left(m_{k} b_{n-k} u-m_{k} b_{n+1-k}\right)\right) \\
& \left(\mathrm{because} b_{0}=1\right) \\
= & \frac{1}{n+1}\left(\left(b_{n}-\sum_{k=1}^{n} m_{k} b_{n-k}\right) u+\left(m_{n+1}+\sum_{k=1}^{n} m_{k} b_{n+1-k}\right)\right) \\
= & b_{n} u+\frac{1}{n+1} \sum_{k=1}^{n+1} m_{k} b_{n+1-k},
\end{aligned}
$$


which leads to:

$$
b_{n+1}=-\frac{1}{n+1} \sum_{k=1}^{n+1} m_{k} b_{n+1-k} .
$$

By induction the formulas hold for all $k=1, \ldots, K$.

Lemma 3.1 is the key to derive the simplified algorithm in the even case. Let $\vec{a}=\left(a_{1}, a_{2}, \ldots, a_{K}\right)^{T}, \vec{b}=\left(b_{1}, b_{2}, \ldots, b_{K}\right)^{T}$; they satisfy the triangular linear systems of equations $A \vec{a}=\vec{m}, B \vec{b}=-\vec{m}$, where

$$
A=\left(\begin{array}{cccc}
1 & 0 & \cdots & \\
-m_{1} & 2 & 0 & \cdots \\
-m_{2} & -m_{1} & 3 & 0 \\
\vdots & \vdots & \ddots & \ddots \\
-m_{K-1} & -m_{K-2} & \cdots & -m_{1} K
\end{array}\right), \quad B=\left(\begin{array}{cccc}
1 & 0 & \cdots & \\
m_{1} & 2 & 0 & \cdots \\
m_{2} & m_{1} & 3 & 0 \\
\vdots & \vdots & \ddots & \ddots \\
m_{K-1} & m_{K-2} & \cdots & m_{1} K
\end{array}\right) .
$$

Note that these matrices are well-conditioned with bounded positive diagonal elements and can be easily solved by standard back substitution. From $\vec{a}$ one next builds the halfsize matrix $\mathbf{P}(u)=A_{1} u-A_{2} \in \mathbb{R}^{K / 2 \times K / 2}$ with

$$
A_{1}=\left(\begin{array}{cccc}
a_{1} & a_{2} & \cdots & a_{K / 2} \\
a_{2} & a_{3} & \cdots & a_{1+K / 2} \\
\vdots & \vdots & \ddots & \vdots \\
a_{K / 2} & a_{1+K / 2} & \cdots & a_{K-1}
\end{array}\right), \quad A_{2}=\left(\begin{array}{cccc}
a_{2} & a_{3} & \cdots & a_{1+K / 2} \\
a_{3} & a_{4} & \cdots & a_{2+K / 2} \\
\vdots & \vdots & \ddots & \vdots \\
a_{1+K / 2} & a_{2+K / 2} & \cdots & a_{K}
\end{array}\right) .
$$

Similarly, one constructs $\mathbf{Q}(u)=B_{1} u-B_{2}$ from $\vec{b}$. Finally, $\Pi(u)=\operatorname{det}(\mathbf{P}) \cdot \operatorname{det}(\mathbf{Q})(u)=$ 0 as soon as

$$
\operatorname{det}\left(A_{1} u-A_{2}\right)=0, \quad \text { or } \quad \operatorname{det}\left(B_{1} u-B_{2}\right)=0 .
$$

In other words, $\vec{u}$ is the set of eigenvalues of $A_{1}^{-1} A_{2}$ and $B_{1}^{-1} B_{2}$. Those can be computed in stable fashion with the QZ-algorithm, available in e.g. the MATLAB or SCILAB packages.

3.3. The odd case: $K \in 2 \mathbb{N}+1$. The algorithm presented in $\S 3.2$ is very convenient to solve Markov's moment problem in case $K \in 2 \mathbb{N}$; unfortunately, geometric optics computations ask more for the opposite case $K \in 2 \mathbb{N}+1$. It turns out that the aforementioned procedure extends to the odd case with minor modifications as we show now.

We first recall that $m_{k}=\sum_{\ell=1}^{K}(-1)^{\ell-1} u_{\ell}^{k}$. Here we let $x_{\ell}$ represent the odd $u_{\ell^{-}}$ values and $y_{\ell}$ the even ones. Since we assume that $K \in 2 \mathbb{N}+1$, we get

$$
m_{k}=\sum_{\ell=1}^{n} x_{\ell}^{k}-\sum_{\ell=1}^{n-1} y_{\ell}^{k}, \quad k=1,2, \ldots, K=2 n-1 .
$$

As previously in (3.3), we solve $B \vec{b}=-\vec{m}$ to get $\vec{b} \in \mathbb{R}^{K}$ and we construct the $(n-1) \times$ $(n-1)$ matrices:

$$
B_{1}=\left(\begin{array}{cccc}
b_{2} & b_{3} & \cdots & b_{n} \\
b_{3} & b_{4} & \cdots & b_{1+n} \\
\vdots & \vdots & \ddots & \vdots \\
b_{n} & b_{1+n} & \cdots & b_{2 n-2}
\end{array}\right), \quad B_{2}=\left(\begin{array}{cccc}
b_{3} & b_{4} & \cdots & b_{1+n} \\
b_{4} & b_{5} & \cdots & b_{2+n} \\
\vdots & \vdots & \ddots & \vdots \\
b_{1+n} & b_{2+n} & \cdots & b_{2 n-1}
\end{array}\right)
$$


Then $\vec{y} \in \mathbb{R}^{n-1}$ is the set of eigenvalues of $B_{1}^{-1} B_{2}$. Now, a set of $2 n$ modified moments can be computed:

$$
\tilde{m}_{0}=n, \quad \tilde{m}_{i}=m_{i}+\sum_{\ell=1}^{n-1} y_{\ell}^{i}=\sum_{\ell=1}^{n} x_{\ell}^{i}, \quad i=1, \ldots, K=2 n-1 .
$$

One can now construct the two $n \times n$ matrices,

$$
A_{1}=\left(\begin{array}{cccc}
\tilde{m}_{0} & \tilde{m}_{1} & \cdots & \tilde{m}_{n-1} \\
\tilde{m}_{1} & \tilde{m}_{2} & \cdots & \tilde{m}_{n} \\
\vdots & \vdots & \ddots & \vdots \\
\tilde{m}_{n-1} & \tilde{m}_{n} & \cdots & \tilde{m}_{2 n-2}
\end{array}\right), \quad A_{2}=\left(\begin{array}{cccc}
\tilde{m}_{1} & \tilde{m}_{2} & \cdots & \tilde{m}_{n} \\
\tilde{m}_{2} & \tilde{m}_{3} & \cdots & \tilde{m}_{1+n} \\
\vdots & \vdots & \ddots & \vdots \\
\tilde{m}_{n} & \tilde{m}_{1+n} & \cdots & \tilde{m}_{2 n-1}
\end{array}\right)
$$

and $\vec{x} \in \mathbb{R}^{n}$ will be the set of eigenvalues of $A_{1}^{-1} A_{2}$. Finally, $\vec{u}=\vec{x} \cup \vec{y}$.

\section{About Wigner measures and Haussdorf's moment problem}

4.1. Wigner transforms and semiclassical limit $\varepsilon \rightarrow 0$. A convenient tool to investigate theoretically high-frequency asymptotics is the Wigner transform, [41]; in the context of (1.1), it reads

$$
W^{\varepsilon}(\psi)(t, x, \xi)=\int_{\mathbb{R}} \psi\left(t, x+\frac{\varepsilon y}{2}\right) \overline{\psi\left(t, x-\frac{\varepsilon y}{2}\right)} \exp (-i \xi y) \cdot d y,
$$

and solves the well-known Wigner equation for all $\varepsilon \geq 0$ :

$$
\partial_{t} W^{\varepsilon}+\xi \partial_{x} W^{\varepsilon}=0 .
$$

If the initial data $W^{\varepsilon}\left(\psi_{0}\right)$ is given by (1.2) then it converges weakly as a measure towards $w(0, x, \xi)=A_{0}^{2}(x) \delta\left(\xi-u_{0}(x)\right)\left(\delta(\cdot)\right.$ the Dirac measure, $\left.u_{0}=\partial_{x} \varphi_{0}\right)$ when $\varepsilon \rightarrow 0$. The limiting solution $w(t, x, \xi)$ also satisfies (4.1). At this level, a classical computation by bicharacteristics implies that (in general), the solution $w(t, x, \xi)$ will split into several branches

$$
w(t, x, \xi)=\sum_{k=1}^{K(t)} \rho_{k}(t, x) \delta\left(\xi-u_{k}(t, x)\right), \quad \sum_{k=1}^{K(t)} \rho_{k}(t, x)=\mathcal{A}^{2}(t, x),
$$

where $\mathcal{A}^{2}$ is the weak limit of $|\psi|^{2}(t, x)$ as $\varepsilon \rightarrow 0$. When there is only one branch, $K(t)=1$, then $\mathcal{A}^{2}=A^{2}$, with $A$ being the amplitude of the WKB signal (1.2) in the $\varepsilon=0$ limit. This is also consistent with a stationary phase analysis for (1.1)-(1.2) as shown in [21].

Following the ideas of $[11,17,23,31,46,49]$, one can consider the moments of the free transport equation satisfied by $w(t, x, \xi)$ with the closure assumption (4.2) for some fixed value $K(t) \equiv K \in \mathbb{N}$. The resulting moment systems are $2 K \times 2 K$ and much more singular ("weakly hyperbolic" in the terminology of [31]):

$$
\partial_{t} \vec{p}+\partial_{x} G_{K}(\vec{p})=0,
$$

where

$$
\vec{p}=\left(\begin{array}{c}
p_{1} \\
\vdots \\
p_{K}
\end{array}\right), \quad G_{K}(\vec{m})=\left(\begin{array}{c}
p_{2} \\
\vdots \\
p_{K+1}
\end{array}\right), \quad p_{j}=\int_{\mathbb{R}} \xi^{j-1} w(t, x, \xi) d \xi=\sum_{k=1}^{K} \rho_{k} u_{k}^{j-1} .
$$


As in Sec. 1.1 these equations follow from multiplying (4.1) by $\xi^{j}$, integrating in $\xi$ over $\mathbb{R}$ and using the closure assumption (4.2). Also in this case, there is no closed form expression for the last component of $G_{K}(\vec{p})$ in terms of $\vec{p}$, except for small values of $K,[23,31,46]$. In order to evaluate $G_{K}$ one is instead led to first solving a delicate moment inversion problem

$$
\text { find }(\vec{\rho}, \vec{u}) \in \mathbb{R}_{+}^{K} \times \mathbb{R}^{K} \quad \text { such that } \sum_{k=1}^{K} \rho_{k} u_{k}^{i}=p_{i+1} ; \quad i=0, \ldots, 2 K-1
$$

and then compute the last component as $\left(G_{K}\right)_{2 K}=\sum_{k=1}^{K} \rho_{k} u_{k}^{2 K}$.

The main difference between these equations and the equations in Sec. 1.1 is that the intensity $A^{2}$ is now directly computed through the $\rho_{k}$ variables as shown in (4.2), instead of via the formula (1.6).

4.2. Stieltje's algorithm for the Dirac closure. The inverse problem (4.3) is well-known and related to Gauss' quadrature rules, [19]; it has been studied by several authors after Chebyshev and Markov, among them we quote [50, 28, 29, 43, 39]. As explained in e.g. [28, 43], the inversion problem (4.3) can be explicitly solved by means of the so-called Stieltje's algorithm which we briefly explain here.

Let us suppose that the moment vector $\vec{p}$ is given. For any polynomial $q(x)$ of degree $n$ less than $2 K$ we define $\mathcal{P}(q)$ as follows

$$
\mathcal{P}(q)=\sum_{i=0}^{n} c_{i} p_{i+1}, \quad q(x)=\sum_{i=0}^{n} c_{i} x^{i} .
$$

We set $q_{-1}(x)=0, q_{0}(x)=1$ and then compute recursively for $k=1, \ldots, K-1$,

$$
q_{k+1}(x)=\left(x-\alpha_{k}\right) q_{k}(x)-\beta_{k-1} q_{k-1}(x),
$$

where

$$
\alpha_{k}=\frac{\mathcal{P}\left(x q_{k}^{2}\right)}{\mathcal{P}\left(q_{k}^{2}\right)}, \quad \beta_{k}=\frac{\mathcal{P}\left(q_{k+1}^{2}\right)}{\mathcal{P}\left(q_{k}^{2}\right)} .
$$

From these numbers we form the tridiagonal matrix $J \in \mathbb{R}^{K \times K}$,

$$
J=\left(\begin{array}{ccccc}
\alpha_{0} & \sqrt{\beta_{0}} & & & \\
\sqrt{\beta_{0}} & \alpha_{1} & \sqrt{\beta_{1}} & & \\
& \ddots & \ddots & \ddots & \\
& & \sqrt{\beta_{K-3}} & \alpha_{K-2} & \sqrt{\beta_{K-2}} \\
& & & \sqrt{\beta_{K-2}} & \alpha_{K-1}
\end{array}\right) .
$$

The unknowns $\left\{u_{k}\right\}$ are now given as the eigenvalues of $J$, and if $\vec{v}_{k}=\left(v_{k, 1}, \ldots, v_{k, K}\right)^{T}$ is a normalized eigenvector corresponding to $u_{k}$, then $\rho_{k}$ is given by the formula

$$
\rho_{k}=p_{1} v_{k, 1}^{2} \text {. }
$$

Unfortunately, the Stieltje's algorithm is known to be unstable when the $\left\{u_{k}\right\}$ get close to each other: $u_{k} \approx u_{\ell}$ for some $k, \ell$. This is a common situation in the semiclassical compuations, e.g. happening when the solution has fewer branches than the PDE supports. The stability for the full problem of computing $\left(G_{K}\right)_{2 K}$ is better, 
but noise coming from the (big) truncation errors inherent in the discretization of the weakly hyperbolic moment systems, $[8,31]$, may still be sufficiently amplified to destabilize the numerical process. At this point some new ideas on regularization of the inversion seem necessary if the method should be used for large $K$. (See below for an example with $K=3$ though.)

\section{tions}

5. Application of Markov's and Stieltje's inversion to WKB computa-

Following [12], we tried to check out a weak consistency of our WKB approximations as $\varepsilon \rightarrow 0$ by looking at the antiderivative of the difference of the position densities (the first quadratic observable); thus we shall study the function

$$
x \mapsto \int_{0}^{x}\left(\rho_{\mathrm{WKB}}(T, s)-\left|\psi^{\varepsilon}(T, s)\right|^{2}\right) \cdot d s,
$$

which can be expected to flatten as $\varepsilon$ is decreased. $\rho_{\mathrm{WKB}}$ stands for the position density obtained from the WKB ansatz (1.2); Lemma 2.1 in [12] ensures that the $L^{1}$ norm of (5.1) going to zero is equivalent to the weak convergence of $\rho_{\mathrm{WKB}}$.

We also wish to make precise the way one can reconstruct a single valued quadratic observable starting from the $\mathbb{R}^{K}$ vectors $\vec{u}$, and $\vec{\mu}$ (we drop the space-time variables for clarity). This is explained in [24] for $K=3$, but more complex cases may arise now. So one can start with some $K$-branch solution $\vec{u} \in \mathbb{R}^{K}$ and look for "jumps" in the $k$ variable, i.e. values $\tilde{k}$ such that,

$$
u_{\tilde{k}-1}-u_{\tilde{k}}>\eta \frac{u_{1}-u_{K}}{K}, \quad \eta=\frac{5}{2} .
$$

(The value $5 / 2$ being arbitrary.) Obviously, the set $K_{J}$ of indices $\tilde{k}$ is included in $\{2, \ldots, K\}$ and the density $\rho_{\mathrm{WKB}}$ reads:

$$
\rho_{\mathrm{WKB}}=\sum_{j=1}^{\left|K_{J}\right|} \sum_{\ell=\tilde{k}_{j-1}}^{\tilde{k}_{j}} \frac{\mu_{\ell}}{\tilde{k}_{j}-\tilde{k}_{j-1}}, \quad \tilde{k}_{0}=1 .
$$

Finally, we initialize the $K$ phases as explained in [20, 24]; we observed that in the mono-valued regions, the thickness $\left(u_{1}-u_{K}\right) / K$ does depend much more on the numerical viscosity on the selected scheme than on its initialization.

5.1. The double cusp interaction: $K=5$. This is the first case for which the exact formulas of [46] don't apply any more. One important point to check is whether the inversion algorithm in $\S 3.3$ remains stable despite the noise generated by the truncation errors in the discretization of the moment systems. The initial data are:

$$
u_{0}(x)=\sin ^{3}(x), \quad \mu_{0}(x)=\frac{1}{\pi} \exp \left(-(x-\pi)^{2}\right), \quad x \in[0,2 \pi] .
$$

The ray geometry is displayed in Fig. 5.1; it shows the superposition of two cusp singularities around $t \simeq 1.5$. In Fig. 5.2, we present the outcome of a "local LaxFriedrichs" scheme called the Rusanov scheme in [7] (as already used in [23, 24]) with $K=5$ at time $t=3$. A comparison with a direct Fourier computation on Schrödinger equation with 4096 modes is also presented for both $\varepsilon=1 / 150$ and $\varepsilon=1 / 450$. The parameters were given by $\Delta x=2 \pi / 1024$ and a CFL number 1 ; the results can be considered as quite satisfying. 


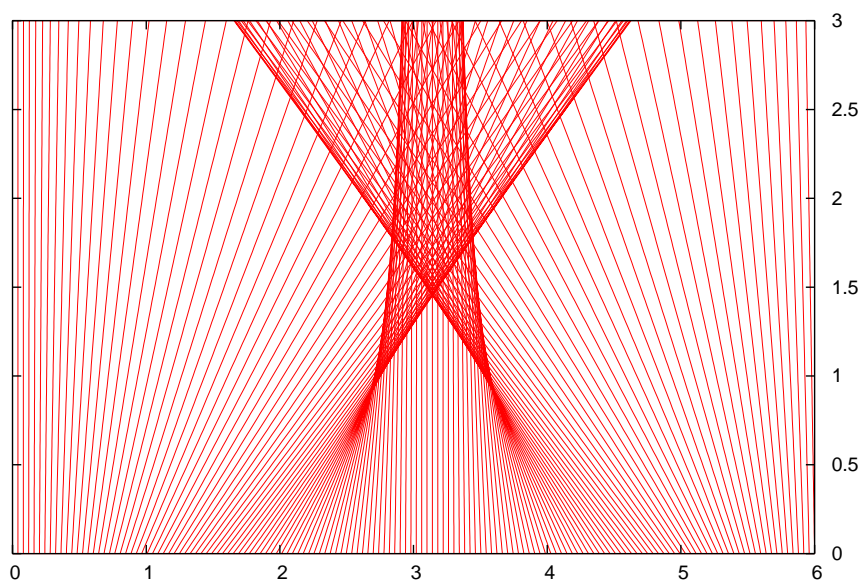

FIG. 5.1. Ray geometry for (5.2) with $t \in[0,3]$.
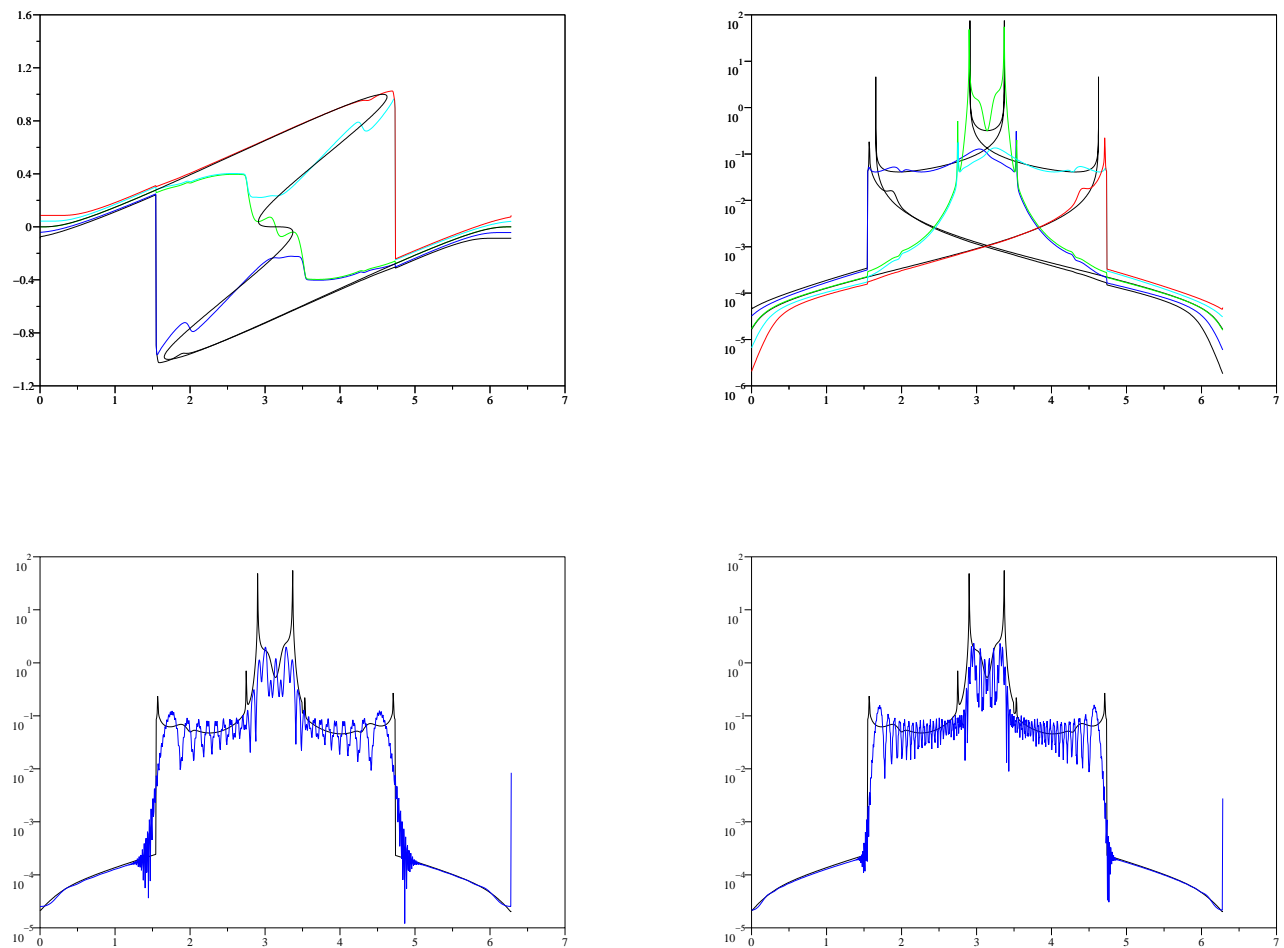

FIG. 5.2. 5-branch solutions and related intensities for (5.2) at $t=3$ (top, solid line is exact solution); comparison of position densities for $\varepsilon=1 / 150,1 / 450$ (bottom, left to right). 


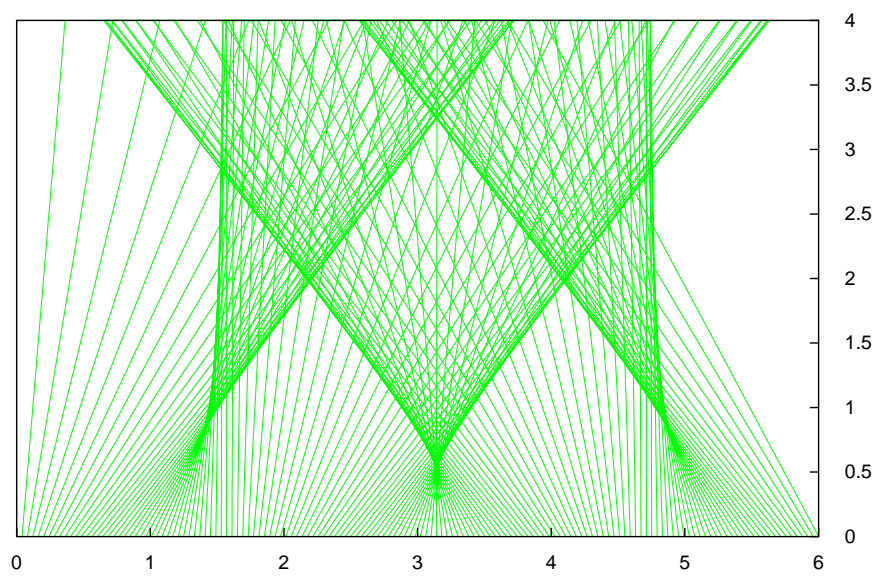

FIG. 5.3. Ray geometry for (5.3) with $t \in[0,4]$.
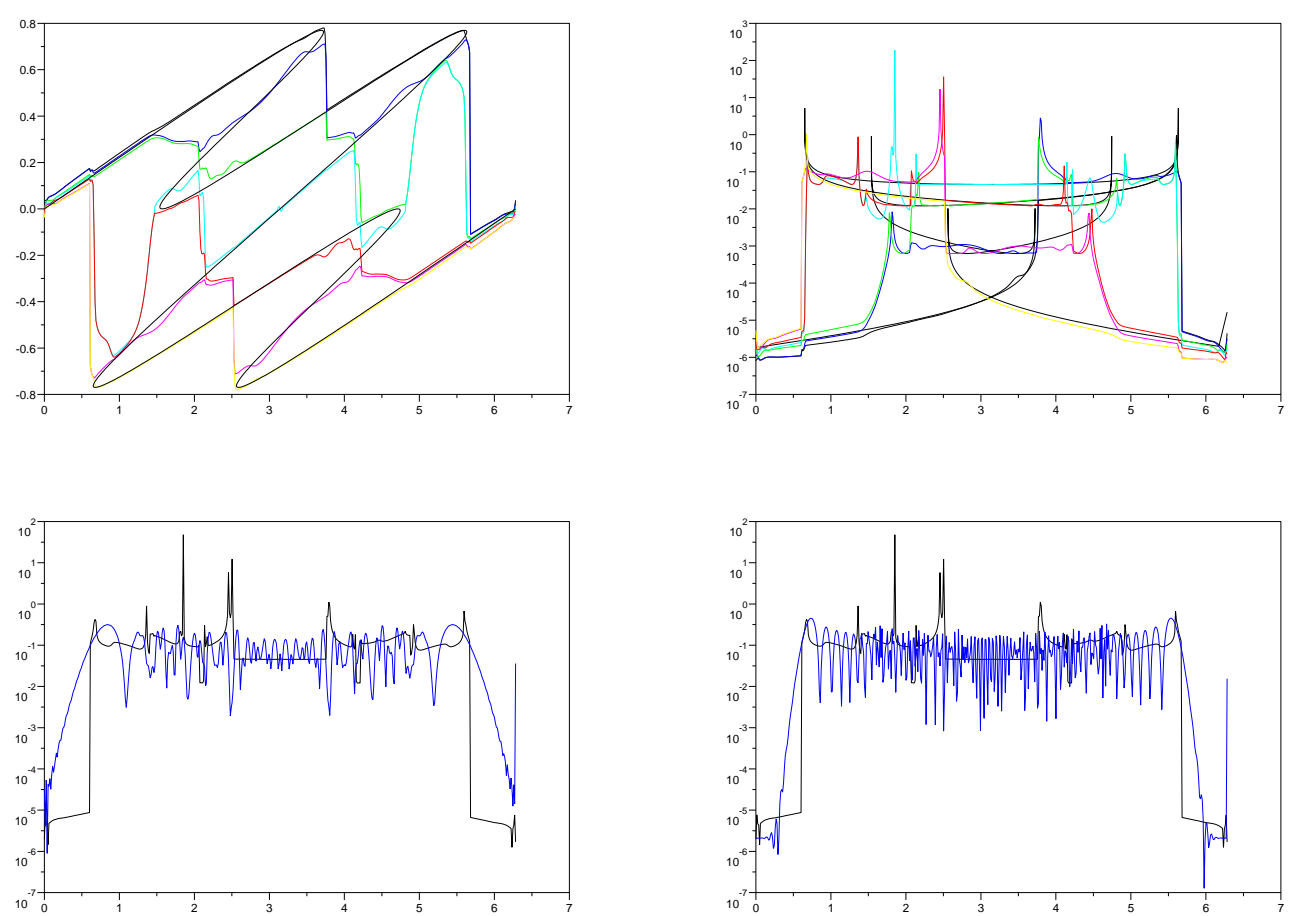

FIG. 5.4. 7-branch solutions and related intensities for (5.3) at $t=4$ (top); comparison of position densities for $\varepsilon=1 / 150,1 / 450$ (bottom). 
The standard CFL condition is based on the fastest waves and creates an excessive numerical dissipation on the slower ones which carry higher intensity (as discussed in [7] pp.32-33). Such a phenomenon clearly induces a bottleneck of performance when trying to reproduce more intricate signals as the dimension of the moment systems $K \times K$ grows, despite our inversion algorithm remaining stable.

In Fig. 5.8 the same problem was solved with the Delta-closure equations and the Stieltje's algorithm. Here only $K=3$ was used to get a stable solution. Then the equations cannot capture all the intricate details of the real wave field, but the qualitative result is correct, including the location of the outermost caustics.

5.2. The triple cusp interaction: $K=7$. We move on to more intricate wave phenomena with the interaction of three cusp singularities: initial data are:

$$
u_{0}(x)=\cos (x) \sin (2 x), \quad \mu_{0}(x)=\frac{1}{\pi} \exp \left(-(x-\pi)^{2}\right), \quad x \in[0,2 \pi] .
$$

The rays show up on Fig.5.3. Our objective is to investigate the reliability of the " $K$-branch solutions" obtained by means of the inversion algorithm presented in $\S 3.3$ for $K$ moderately big and to compare the first quadratic observable (the position density in the context of Schrödinger equation (1.1)) deduced this way with the one coming out of a direct Fourier computation. The present test case aims at exploiting the $K=7$ closure; the numerical results are presented on Fig.5.4. One can see a very satisfying picture, since the velocities are well rendered and the approximation of the position densities are very acceptable except for the caustics.

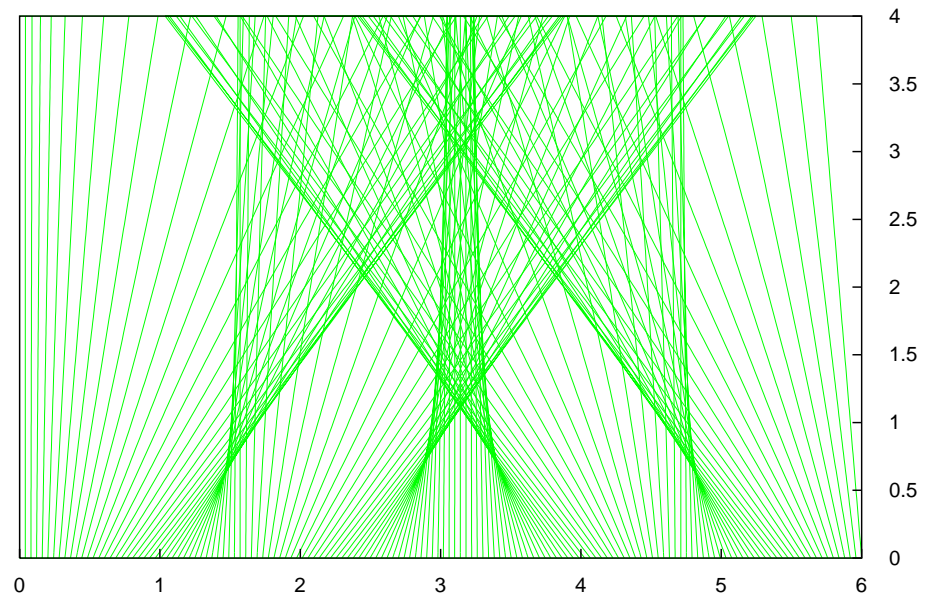

FIG. 5.5. Ray geometry for (5.4) with $t \in[0,4]$.

5.3. Four superimposing cusps: $K=7$ or $K=9$ ? We now study what happens for many cusp interactions as time increases:

$$
u_{0}(x)=\sin ^{2}(2 x) \sin (x), \quad \mu_{0}(x)=\frac{1}{\pi} \exp \left(-(x-\pi)^{2}\right), \quad x \in[0,2 \pi] .
$$



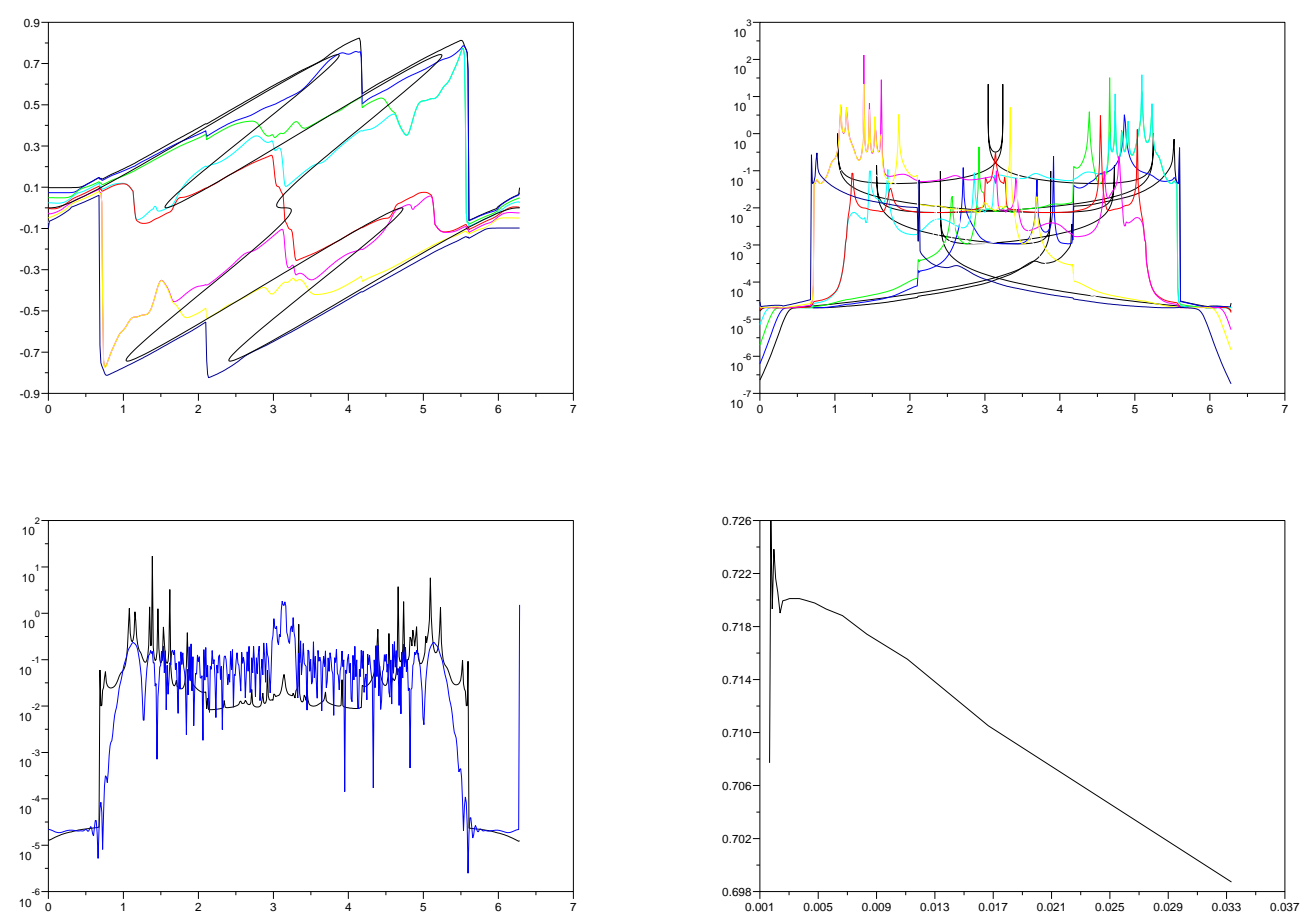

FIG. 5.6. 9-branch solutions and related intensities for (5.4) at $t=4$ (top); comparison of position densities for $\varepsilon=1 / 450$ and evolution of the $L^{1}$ norm of (5.1) as $\varepsilon \rightarrow 0$ (bottom).

At time $t=4$, the choice $K=9$ appears to be necessary and one observes a general numerical discrepancy in the whole computational domain since many phases come into play. The comparison with a direct Schrödinger result is less significant; this is a consequence of the numerical viscosity of the marching schemes we used for solving the moment system as explained in $\$ 5.1$. The use of more sophisticated algorithms, like e.g. $[1,2,14]$ provided only marginal improvements in this context, with a lower CFL number whereas the HLL or kinetic solvers written in [7] led to better results.

Looking at Fig.5.6, one may think that discarding the multivaluations close to $x=\pi$ can be a reasonable choice and decide to work out (5.4) with the $K=7$ closure; the outcome is shown in Fig.5.7. Despite the fact it does not seem too different compared to Fig.5.6, it contains a compressive Lax shock and this should create an intensity concentration in $x=\pi$. Of course, the errors as measured with the $L^{1}$ norm of (5.1) as $\varepsilon \rightarrow 0$ are bigger when compared to the ones of the 9-branch computation, see Fig.5.6. It would also be possible in this context to exploit the "symmetry trick" with the $K=5$ closure as introduced in [20], but it is not our purpose here.

\section{Conclusion and outlook}

This work is merely a first step towards the development of efficient moment-based algorithms to treat one-dimensional or two-dimensional paraxial geometric optics and semiclassical limits in a general multivalued context. We restricted ourselves to the free Schrödinger equation (1.1) for simplicity only, but external potentials can be 

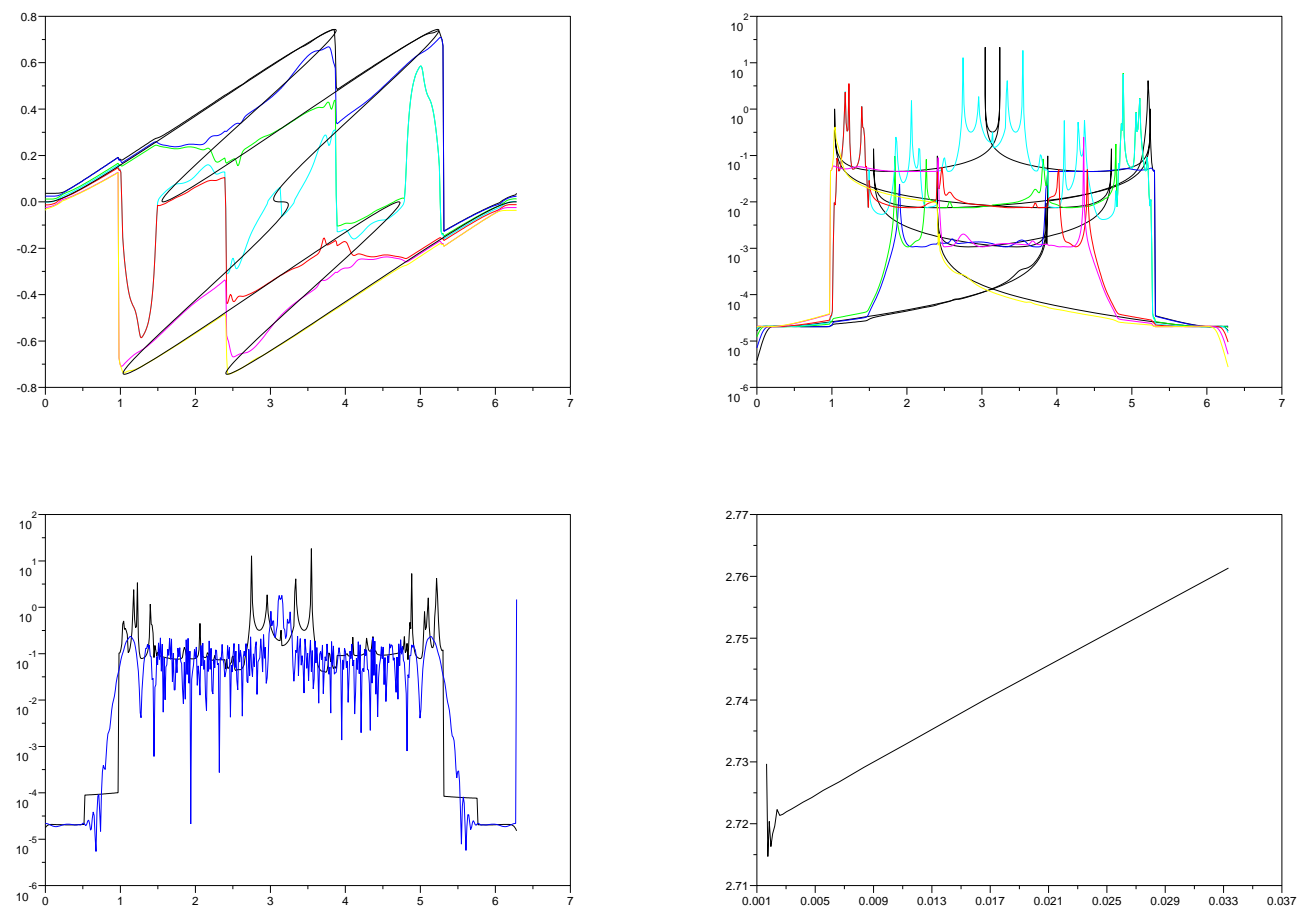

FIG. 5.7. 7-branch solutions and related intensities for (5.4) at $t=4$ (top); comparison of position densities for $\varepsilon=1 / 450$ and decay of the $L^{1}$ norm of (5.1) as $\varepsilon \rightarrow 0$ (bottom).
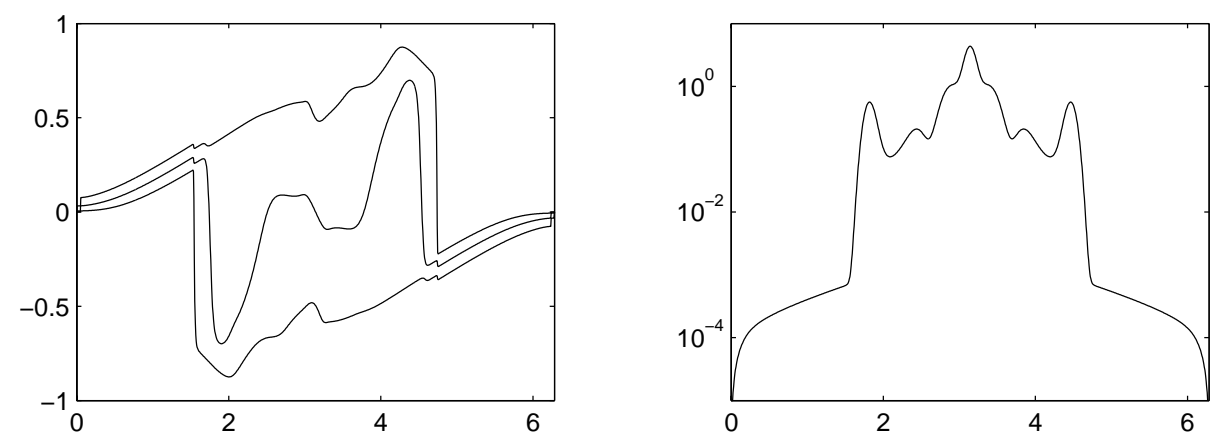

FIG. 5.8. 3-valued solutions and related intensities for (5.2) at $t=3$ computed with the Delta equations and Stieltje's algorithm.

included, as in [20, 23]. Applications to solid-state physics, where an oscillating potential has to be taken care of, is possible following the ideas of [24]. Trigonometric moment problems have to be tackled when considering geometric optics problems, following $[46,20,22]$ and the techniques of $[13,35,48]$.

Most of all, it appeared that an important step will be the development of efficient 
numerical schemes for the simulation of the nonstrictly hyperbolic moment systems (1.10) which can render the slow (and more energetic!) waves in a correct way. Up to now, it does not seem that usual discretizations can achieve this goal. See [7] for a recent summary. At last, we emphasize that inverting moments of the type (1.10) is a task which has to be achieved inside each computational cell, since it is necessary in order to compute any kind of numerical flux; it is somehow part of the derivation of these numerical fluxes.

Acknowledgement. Support by the Wolfgang Pauli Institute (Wien) in the frame of the "Nanoscience" Programme and the European network HYKE, funded by the EC as contract HPRN-CT-2002-00282 is acknowledged.

\section{REFERENCES}

[1] Robert Artebrant, Limiter-free third order logarithmic reconstruction, SIAM J. Sci. Comp., to appear.

[2] Robert Artebrant and Hans Joachim Schroll, Conservative logarithmic reconstructions and finite volume methods, SIAM J. Sci. Comp., to appear.

[3] J. D. Benamou, Direct computation of multivalued phase space solutions for Hamilton-Jacobi equations, Comm. Pure Appl. Math., 52, 1443-1475, 1999.

[4] F. A. Bornemann, P. Nettescheim and C. Schütte, Quantum-Classical Molecular Dynamics as an approximation to full quantum dynamics, J. Chem. Phys., 105, 1074-7083, 1996.

[5] J. M. Borwein and A. S. Lewis, Moment-matching and best entropy estimation, J. Math. Anal. Appl., 185, 596-604, 1994.

[6] J. M. Borwein, A. S. Lewis and M. A. Limber, Entropy minimization with lattice bounds, J. Approx. Theory, 79, 1-16, 1994.

[7] F. Bouchut, Nonlinear stability of finite volume methods for hyperbolic conservation laws, and well-balanced schemes for sources, Frontiers in Mathematics series, Birkhäuser, ISBN 3-76436665-6, 2004

[8] F. Bouchut, S. Jin and X. Li, Numerical approximations of pressureless and isothermal gas dynamics, SIAM J. Numer. Anal., 41, 135-158, 2003.

[9] Y. Brenier, Averaged multivalued solutions for scalar conservation laws, SIAM J. Numer. Anal., 21, 1013-1037, 1984

[10] Y. Brenier, Équations de moment et conditions d'entropie pour des modèles cinétiques, (French), (Moment equations and entropy conditions for kinetic models), Séminaire sur les Équations aux Dérivées Partielles, 1994-1995, Exp. No. XXII, 11, École Polytech., Palaiseau, 1995.

[11] Y. Brenier and L. Corrias, A kinetic formulation for multibranch entropy solutions of scalar conservation laws, Ann. I. H. P. Nonlinear Anal., 15, 169-190, 1998.

[12] Y. Brenier and E. Grenier, Sticky particles and scalar conservation laws, SIAM J. Num. Anal., 38, 2317-2328, 1998.

[13] Th. Chonavel and Ph. Loubaton, Le problème des moments trigonométriques sur un intervalle, C. R. Acad. Sc., 314, 41-44, 1992.

[14] P. Degond, P. F. Peyrard, G. Russo and P. Villedieu, Polynomial upwind schemes for hyperbolic systems, C. R. Acad. Sci. Paris Ser. I Math., 328, 479-483, 1999.

[15] P. Diaconis and D. Friedman, The Markov moment problem and de Finetti's theorem, Math. Zeitschrift, to appear.

[16] B. Engquist and O. Runborg, Computational high frequency wave propagation, Acta Numerica, 12, 181-266, 2003.

[17] S. Filippas and G. N. Makrakis Semiclassical Wigner functions and geometrical optics, SIAM Multiscale Modeling \& Simulation, 1, 4, 674-710, 2004

[18] I. Gasser, C. K. Lin and P. A. Markowich, A review of dispersive limits of (non)linear Schrödinger-type equations, Taiwanese J. Math., 501-529, 2000.

[19] W. Gautschi, Moments in quadrature problems, Comput. Math. Appl. 33, 105-118, 1997.

[20] L. Gosse, Using K-branch entropy solutions for multivalued geometric optics computations, J. Comp. Phys., 180, 155-182, 2002.

[21] L. Gosse, A case study on the reliability of multiphase WKB approximation for the onedimensional Schrödinger equation, IRMA Lectures Notes 7, 151-161, 2005.

$[22]$ L. Gosse and F. James, Convergence results for an inhomogeneous system arising in various high frequency approximations, Numer. Math., 90, 721-753, 2002. 
[23] L. Gosse, S. Jin and X. Li, Two moment systems for computing multiphase semiclassical limits of the Schrödinger equation, Math. Models Meth. Appl. Sci., 13, 1689-1723, 2003.

[24] L. Gosse and P. A. Markowich, Multiphase semiclassical approximation of an electron in a onedimensional crystalline lattice - I. Homogeneous problems., J. Comp. Phys., 197, 387-417, 2004.

[25] L. Gosse and O. Runborg, Numerical inversion of the min-power moment system, In preparation, 2004.

[26] E. Grenier, Semiclassical limit of the nonlinear Schrödinger equation in small time, Proc. Amer. Math. Soc., 126, 523-530, 1998.

[27] M. Gugat, Time-Optimal Control: Algorithms Based Upon Moment Equations and Parametric Programming, Habilitationsschrift im Fachbereich IV der Universitat Trier, 1999. http://www.mathematik.tu-darmstadt.de/ gugat/

[28] G. Inglese, A note about the discretization of finite moment problems, Inverse Problems, 10, 401-414, 1994.

[29] G. Inglese, Christoffel functions and finite moment problems, Inverse Problems, 11, 949-960, 1995.

[30] S. Izumiya and G. T. Kossioris, Geometric singularities for solutions of single conservation laws, Arch. Rational Mech. Anal., 139, 255-290, 1997.

[31] Shi Jin and X. Li, Multi-phase computations of the semiclassical limit of the Schrödinger equation and related problems: Whitham vs. Wigner, Physica D, 182, 46-85, 2003.

[32] S. Jin, H. L. Liu, S. Osher and R. Tsai, Computing multivalued physical observables for the semiclassical limit of the Schrödinger equations, J. Comp. Phys., to appear.

[33] J. B. Keller, Semiclassical mechanics, SIAM Review, 27, 485-504, 1985.

[34] V. I. Korobov and G. M. Sklyar, Time-optimality and the power moment problem, Russian, Mat. Sb. N.S., 134, 176, 186-206, 287, 1987; translation in Math. USSR-Sb., 62, 1, 185-206, 1989.

[35] V. I. Korobov and G. M. Sklyar, Time-optimality and the trigonometric moment problem, Russian, Izv. Akad. Nauk SSSR Ser. Mat., 53, 4, 868-885, 1989; translation in Math. USSRIzv., 35, 1, 203-220, 1990.

[36] V. I. Korobov and G. M. Sklyar, The Markov moment min-problem and time optimality, Russian, Sibirsk. Mat. Zh., 32, 1, 60-71, 220, 1991; translation in Siberian Math. J., 32, 1, 46-55, 1991.

[37] G. T. Kossioris, Formation of singularities for viscosity solutions of Hamilton-Jacobi equations in one space varible Comm. P. D. E., 18, 747-770, 1993.

[38] M. G. Krein and A. A. Nudel'man, The Markov moment problem and extremal problems, A. M. S. translations, 1977.

[39] M. J. Leaseburg and L. R. Mead, Error bounds in maximum entropy approximations, J. Math. Phys., 34, 6009-6015, 1993.

[40] A. S. Lewis, Superresolution in the Markov moment problem, J. Math. Anal. Appl., 197, 774$780,1996$.

[41] P. L. Lions and T. Paul, Sur les measures de Wigner, Revista Mat. Iberoamericana, 9, 553-618, 1993.

[42] P. A. Markowich, P. Pietra and C. Pohl, Numerical approximation of quadratic observables of Schrödinger-type equations in the semiclassical limit, Numer. Math., 81, 595-630, 1999.

[43] L. R. Mead and N. Papanicolaou, Maximum entropy in the problem of moments, J. Math. Phys., 25, 2404-2417, 1984.

[44] D. T. Norris, Optimal solutions to the $L^{\infty}$ moment problem with lattice bounds, Ph. D. thesis, Univ. Colorado, 2002. http://math.colorado.edu/ norrisdt/dougthesis.ps

[45] J. Qian and S. Leung, A level-set based Eulerian method for paraxial multivalued traveltimes, J. Comp. Phys., 197, 711-736, 2004.

[46] O. Runborg, Some new results in multiphase geometrical optics, Math. Mod. Numer. Anal., 34 , 1203-1231, 2000.

[47] G. M. Sklyar and L. V. Fardigola, The Markov power moment problem in problems of controllability and frequency extinguishing for the wave equation on a half-axis, J. Math. Anal. Appl., 276, 109-134, 2002.

[48] G. M. Sklyar and L. V. Fardigola, The Markov trigonometric power moment problem in controllability problems for the wave equation on a half-axis, Matem. Fizika, Analiz, Geometria, 9, 233-242, 2002.

[49] C. Sparber, P. Markowich and N. Mauser, Multivalued geometrical optics: wigner functions vs. WKB methods, Asymptotic Anal., 33, 153-187, 2003.

[50] G. Talenti, Recovering a function from a finite number of moments, Inverse Problems, 3, 501$517,1987$. 